\title{
Characterisation of thermally sprayed metallic NiCrAlY deposits by multiple small-angle scattering
}

\author{
T. Keller ${ }^{1, *}$, W. Wagner ${ }^{1}$, A. Allen ${ }^{2}$, J. Ilavsky ${ }^{2}$, N. Margadant ${ }^{3}$, S. Siegmann ${ }^{3}$, G. Kostorz ${ }^{4}$ \\ ${ }^{1}$ Paul Scherrer Institute, 5232 Villigen PSI, Switzerland \\ ${ }^{2}$ National Institute of Standards and Technology, Gaithersburg, MD 20899, USA \\ ${ }^{3}$ Swiss Federal Laboratories for Materials Testing and Research, 3602 Thun, Switzerland \\ ${ }^{4}$ Swiss Federal Institute of Technology, 8093 Zurich, Switzerland \\ Received: 16 July 2001/Accepted: 24 October 2001 - (C) Springer-Verlag 2002
}

\begin{abstract}
The technique of multiple small-angle neutron scattering (MSANS) was applied to thermally sprayed metallic deposits. The samples are technologically relevant $\mathrm{Ni}$ based NiCrAlY coatings manufactured by different spray techniques (atmospheric and water-stabilised plasma spraying, flame spraying). MSANS measurements were made in directions parallel and perpendicular to the surface plane. Based on empirical considerations and on the analysis of SEM micrographs of coating cross-sections, the complex microstructures were modelled in terms of three distinct void systems. Employing a recent MSANS formalism, the experimental beam-broadening as a function of incident wavelength was used to derive the model parameters under the constraints of MSANS anisotropy, total measured porosity, and total internal surface area. The surface area was obtained from smallangle neutron scattering analysis in the Porod regime.
\end{abstract}

PACS: $81.15 . R s ; 61.72 . Q q$

The void structure of thermally sprayed coatings significantly influences the deposit properties [1]. Microstructural investigations, in particular, characterisation of the pore systems contribute to the optimisation of the thermal-spray process. Metallic NiCrAIY deposits $(67 \% \mathrm{Ni}, 22 \% \mathrm{Cr}, 10 \% \mathrm{Al}, 1 \% \mathrm{Y}$, all in mass fraction) have been studied by small-angle neutron scattering (SANS) in the Porod scattering regime to obtain information on the total specific surface area of the voids [2]. By the method of multiple SANS (MSANS) analysis it is possible to model the void structure in more detail, distinguishing distinct subsystems of globular pores and oblate spheroidshaped pores. The model parameters comprise void volume fractions, average sizes and orientation distributions. While several spray techniques have been investigated to compare the influence of the spray process on the void system, the quantitative discussion of the results presented here treats one deposit produced by atmospheric plasma-spray (APS).

${ }^{*}$ Corresponding author. (Fax: +41-56/310-3131, E-mail: t.keller@psi.ch)

\section{Experiment}

The deposits were manufactured using standard sprayprocess parameters with the spray direction perpendicular. to the substrate. The particle size range of the feedstock powder was appropriate for the APS spray technique, as described in detail in [3]. Free-standing deposits were obtained by removing the substrate. From the same sample material, 2.3-mm-thick cross-section samples were prepared by cutting. The cross-section samples were investigated with the spray direction perpendicular to the incident beam direction. The free-standing deposits were aligned with the neutron beam parallel to the spray direction.

The 8-m SANS instrument [4] at the Center for Neutron Research, National Institute of Standards and Technology, Gaithersburg, USA, was used to perform the SANS and the MSANS measurements. The cross-section samples were investigated at a neutron wavelength $(\lambda=8 \AA)$ and a resolution of $\Delta \lambda / \lambda=0.15$. Following the usual data-reduction procedures [4], the experimental data were averaged with regard to the azimuthal angle in $15^{\circ}$-wide sectors around the incident beam direction. The absolute scattering cross-section was obtained as a function of the magnitude of the scattering vector: $Q=4 \pi \sin \theta / \lambda$, where $\theta$ is half the scattering angle. A power law of $Q^{-4}$ was fitted to the data in the Porod regime, and the total specific surface area, $S_{\text {tot }}$, was determined according to

$\langle\mathrm{d} \Sigma(Q) / \mathrm{d} \Omega\rangle=2 \pi|\Delta \varrho|^{2} S_{\mathrm{tot}} / Q^{4}$,

where $\mathrm{d} \Sigma(Q) / \mathrm{d} \Omega$ denotes the absolute macroscopic singlescattering cross-section in each direction of $Q,\langle\cdot\rangle$ an orientational average of the sectors over all azimuthal angles exploiting the axial symmetry of the microstructure about the spray direction, and $|\Delta \varrho|^{2}$ the neutron scattering contrast between the metal matrix and the voids.

The MSANS beam-broadening at wavelengths of 12,13 , $14,15,16,17$ and $18 \AA$ was determined both for the crosssection samples and for the free-standing deposits.

The MSANS beam-broadening is expressed in terms of the radius of curvature, $r_{\mathrm{c}}$, of the scattering curve at 
$Q=0$, and is numerically equal to the standard deviation of a Gaussian fitted to the experimental data. For both sample orientations, the experimental data were azimuthally averaged around the incident beam to yield the MSANS beam-broadening of, effectively, two different axially symmetric microstructures. To obtain the MSANS anisotropy, the MSANS data for the cross-section samples were also sectoraveraged in $15^{\circ}$-wide sectors. An ellipse was fitted to the anisotropic $r_{\mathrm{c}}$ data, and the MSANS anisotropy is expressed in terms of the aspect ratio of the fitted ellipse.

The void structure was modelled to fit the orientationally averaged MSANS beam-broadening $r_{\mathrm{c}}$ data obtained for the two sample orientations, consistent with the total porosity determined from precision density measurements, as described in [3]. The experimentally determined (Porod) total specific surface area and the observed MSANS anisotropy had to be obeyed as constraints for the model fit. For the theoretical basis on the MSANS analysis, as applicable to coatings, we refer the reader to [5]. A quantitative confirmation of the MSANS method by ultrasmall-angle X-ray scattering (USAXS) can be found in [6].

\section{Results}

The total porosity of the APS deposit was previously determined to be 0.081 with a standard deviation of \pm 0.001 [2] From the known chemical composition and density of the alloy, the scattering contrast with the voids was calculated to be $|\Delta \varrho|^{2}=41.34 \times 10^{28} \mathrm{~m}^{-4}$. The total specific surface area from the SANS analysis was found to be $(0.766 \pm$ $0.010) \times 10^{6} \mathrm{~m}^{-1}$. The experimentally determined circularly averaged MSANS beam-broadening is shown in Fig. 1 for the cross-section and free-standing deposit. Model and experimental anisotropies are shown in the polar plot of Fig. 2. The MSANS anisotropy of the cross-section sample, determined by averaging the aspect ratios of the ellipses for all wavelengths, was found experimentally to be $1.35 \pm 0.03$.

The model applied assumes the total void volume fraction to be split into three distinct subsystems: intralamellar cracks comprised of oblate spheroidal elements with

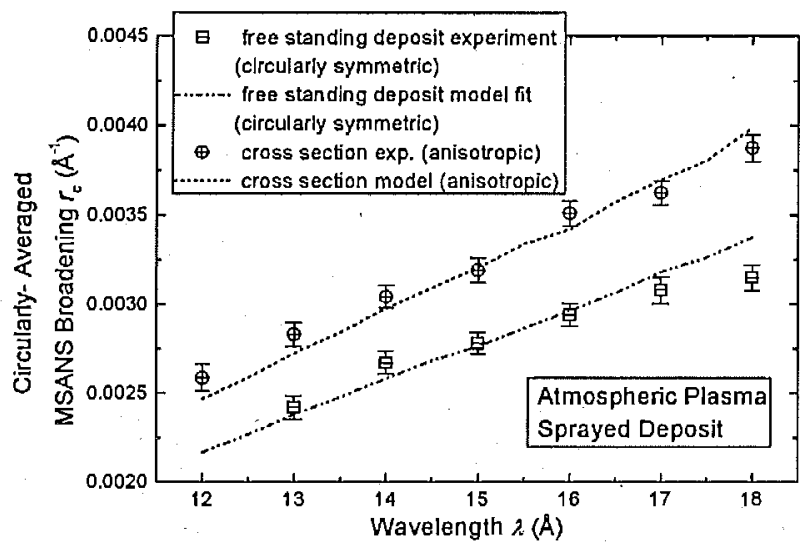

Fig. 1. Experimental data of the circularly averaged MSANS beanbroadening from the APS cross-section and free-standing deposit together with the model fits. Vertical bars represent the standard deviations

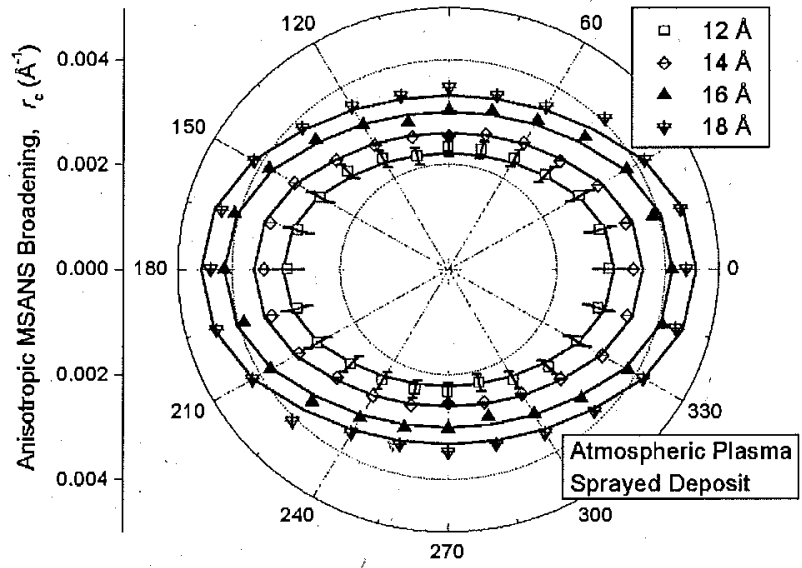

Fig. 2. Anisotropic MSANS beam-broadening as measured and predicted by the model for different wavelengths. Standard deviations of the experimental data are indicated for $\lambda=12 \AA$

their short dimension preferentially aligned perpendicular to the spray direction, interlamellar pores also comprised of oblate spheroidal elements with their short dimension mainly aligned parallel to the spray direction, and globular pores. Aspect ratios of the elements used to model the cracks and interlamellar pores were chosen to be $\beta=1 / 10$ and $\beta=1 / 5$, respectively. To account for the different orientation distributions of the cracks and interlamellar pores, possible orientations are divided, in each case, into 3 ranges with respect to the spray direction: 0 to $30^{\circ}, 30$ to $60^{\circ}$ and 60 to $90^{\circ}$. The probabilities of finding a crack or pore within each of these ranges are given by $g_{L}, g_{M}$, and $g_{H}$, respectively. The model probabilities, used for the APS deposit, are given in Table 1.

The mean sizes of the elements used to model the three void systems were parameterized by the ratios of their large dimension. For the APS deposit modelled here, suitable ratios were found to be: $\langle R\rangle_{\mathrm{P}} /\langle R\rangle_{\mathrm{C}}=1.0$ and $\langle R\rangle_{\mathrm{G}} /\langle R\rangle_{\mathrm{C}}=1.3$, where $C$ stands for cracks, $P$ for interlamellar and $G$ for globular pores. By non-linear least-squares fitting, the average large radius of the cracks, being the only remaining free fitting parameter, was found to be $\langle R\rangle_{\mathrm{C}}=0.728 \pm 0.010 \mu \mathrm{m}$. From $\langle R\rangle_{\mathrm{C}}$, values of $\langle R\rangle_{\mathrm{P}}$ and $\langle R\rangle_{\mathrm{G}}$ were determined. The small radii then are given by $\beta_{\mathrm{C}}\langle R\rangle_{\mathrm{C}}$ and $\beta_{\mathrm{P}}\langle R\rangle_{\mathrm{P}}$. From the model parameters, a distribution of the specific surface area among the three void systems and the mean opening dimensions, defined by $\langle$ O.D. $\rangle=4 \beta\langle R\rangle / 3$ were deduced and are given in Table 2 . The total specific surface area calculated from the model parameters by summing all three components is $S_{\text {tot }}=0.765 \times 10^{6} \mathrm{~m}^{-1}$ which is within the standard deviation of the experimental value for $S_{\text {tot }}$ of $0.766 \times 10^{6} \mathrm{~m}^{-1}$, obtained from neutron scattering in the Porod regime. The model suggests a MSANS anisotropy of 1.38 , which is also in good agreement with the experimental value of 1.35 .

\begin{tabular}{lccc}
\hline & $g_{\mathrm{L}}$ & $g_{\mathrm{M}}$ & $g_{\mathbf{H}}$ \\
\hline Crack system & 0.04 & 0.22 & 0.74 \\
Pore system & 0.64 & 0.31 & 0.05
\end{tabular}

Table 1. Orientation distributions of the intralamellar cracks and interlamellar pore systems derived from the MSANS model for the APS deposit 


\begin{tabular}{lccc}
\hline & $\begin{array}{c}\text { Specific surface } \\
\text { area }\left(10^{6} \mathrm{~m}^{-1}\right)\end{array}$ & $\begin{array}{c}\langle\mathrm{O} . \mathrm{D} .) \\
(\mu \mathrm{m})\end{array}$ & $\begin{array}{c}\text { Mean pore } \\
\text { diameter }(\mu \mathrm{m})\end{array}$ \\
\hline Intralamellar cracks & 0.127 & 0.097 & - \\
Interlamellar pores & 0.563 & 0.194 & - \\
Globular pores & 0.079 & - & $1 . \overline{8} 92$ \\
\hline
\end{tabular}

Table 2. Specific surface areas and mean opening dimensions for the three void subsystems of the APS deposit derived from the MSANS model

\begin{tabular}{lcc}
\hline & $\begin{array}{c}\text { Specific surface } \\
\text { area fraction (\%) }\end{array}$ & $\begin{array}{c}\text { Volume } \\
\text { fraction (\%) }\end{array}$ \\
\hline Intralamellar cracks & 17 & 7 \\
Interlamellar pores & 73 & 62 \\
Globular pores & 10 & 31 \\
\hline$\Sigma_{\text {total }}$ & 100 & 100 \\
\hline
\end{tabular}

Table 3. Specific surface areas and volume fractions of the void subsystems, calculated from the MSANS model parameters

In Table 3 the component-specific surface areas and volume fractions are given. Estimated fractional standard deviations are $\pm 10 \%$ for the component porosities and $\pm 5 \%$ for the orientation probabilities, component surface areas, 〈O.D. values, and the mean globular pore diameter.

\section{Discussion and conclusion}

Since from conventional SANS it is not possible to obtain direct size information from extended microstructures such as thermally sprayed deposits, an MSANS analysis was applied to obtain detailed information from the wavelengthdependent broadening of the primary neutron beam. Under the strict constraints of the independently determined (Porod) total specific surface area, total pore volume fraction and MSANS anisotropy, the void system of a metallic NiCrAlY APS deposit was assigned to three void subsystems, and their mean sizes and orientation distributions were modelled. The model results, suggest only a small contribution to the total void volume fraction from the cracks. This picture appears to be confirmed by the SEM image in Fig. 3, which shows very

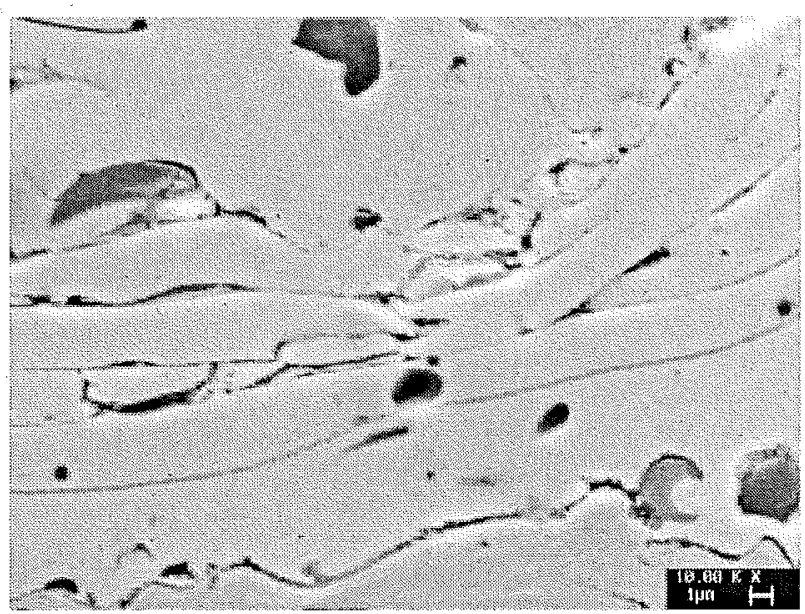

Fig. 3. SEM micrograph of a polished cross-section of the APS deposit. The spray direction is vertical

few cracks. While their volume fraction is only $7 \%$, the elements of the crack systems contribute $17 \%$ to the total specific surface area.

Acknowledgements. We acknowledge the NIST Center for Neutron Research for use of the SANS facility. Part of this work was funded by the Eureka / KTI grant S1973 THERMETCOAT.

\section{References}

1. J. Ilavsky, A.J. Allen, G.G. Long, S. Krueger, C.C. Berndt, H. Herman: J. Am. Ceram. Soc. 80(3), 733 (1997)

2. T. Keller, W. Wagner, J. Ilavsky, N. Margadant, S. Siegmann, J. Pisacka, J. Matejicek, G. Barbezat, P. Fiala, T. Pirling: In: Proc. $2^{\text {nd }}$ Int. Thermal Spray Conference, ed. by C.C. Berndt (ASM International, Materials Park, $\mathrm{OH} 44073-0002$, USA 2001) p. 653

3. N. Margadant, S. Siegmann, J. Patscheider, T. Keller, W. Wagner, J. Ilavsky, J. Pisacka, G. Barbezat, P. Fiala: In: Proc. $2^{\text {nd }}$ Int. Thermal Spray Conference, ed. by C.C. Berndt (ASM International, Materials Park, OH 44073-0002, USA 2001) p. 643

4. C.J. Glinka, J.M. Rowe, J.G. LaRock: J. Appl. Cryst. 19, 427 (1985)

5. A.J. Allen, J. Ilavsky, G.G. Long, J.S. Wallace, C.C. Berndt, H. Herman: Acta Mater: 49, 1661 (2001)

6. G.G. Long, S. Krueger, A.J. Allen: J. Neutron Res. 7, 195 (1999) 